\title{
BANDWIDTH RESERVATION ALGORITHM FOR WIRELESS CELLULAR NETWORKS
}

\author{
I. I. Ibrahim ; A.S. Ali \\ Faculty of Engineering, Helwan University, Cairo, Egypt \\ iiibrahim@softhome.net ; ah_salah@softhome.net
}

\author{
A. F. Ghanim \\ Telecom Egypt , Wireless net. Planning Dept, Cairo, Egypt \\ ahmed.fathy@telecomegypt.com.eg
}

(Received August 10, 2006 Accepted December 24, 2006)

This paper presents an advanced bandwidth reservation technique to improve Quality of Service $(Q o S)$ by reducing the forced call termination during handoff. One solution to ensure continuity of on-going calls and reduce the forced call termination during handoff is to predict Mobile Station (MS) trajectory and do resource reservation in advance. So, we propose a new scheme that uses an accurate mobility prediction algorithm to predict MS next location and uses this data in bandwidth reservation scheme to improve QoS. The performance of the algorithm is evaluated via simulations. The simulation results show that our algorithm reduces handoff call drop rate without over reserving the resources hence it improves bandwidth utilization and reduces new call blocking rate.

\section{INTRODUCTION}

Mobility in wireless networks allows more flexible model of communication, but it also makes problems for network designers to decide the network capacity, handoff, bandwidth reservation and QoS. One of the most important QoS issues is how to control dropping running calls. To reduce the call drop probability the network saves some channels for handoff calls (guarded channel). New calls will be blocked if the number of idle channels is equal to or less than the number of guarded channels, while handoff calls can be served until all the channels are occupied. Many algorithms proposed to give priority for the existing call over the new call using guard channel. Generally there are two strategies: fixed guard channel and dynamic guard channel. In fixed guard channel $[1,2]$ fixed portion of channels is reserved for handoff calls. This will reduces handoff calls dropping probability substantially at the expense of slight increases in new call blocking probability by giving bandwidth access priority to handoff calls over new calls in call admission control. Dynamic guard channel overcomes the shortage of fixed guard channel by dynamically changing the reserved bandwidth for handoff depending on required bandwidth for expected handoff [3 -12]. 
In $[3,11,12]$, Shadow Cluster (SC) algorithm is proposed. It assumes that every active mobile exerts an influence upon the cells in the vicinity of its current location and along its direction of travel. The algorithm estimates the required reserved bandwidth in the cells under SC. Predictive Schemes for Handoff Prioritization [7], propose that based on MS position and orientation the Base Transceiver Station (BTS) estimate MS path. When the MS is within a certain distance (threshold distance) from the next cell it sends channel reservation to next cell. This method was further pursued in [8], but instead of using threshold distance it uses threshold time. In dynamic channel reservation algorithm [5], the guard channel is dynamically changed according to the requested probability which is determined by the mobility patterns of the MS and traffic load. In [6], based on the MS's speed, direction and road information, the BTS estimates the probability that the MS will enter neighbor cell. The BTS then calculate the reserved bandwidth. But the algorithm doesn't determine the time when the MS handoff to and from the cell. Also it assumes that the MS is moving in constant speed during call holding time and all MSs move in roads which are inside the road map and can't move outside the road map.

Many bandwidth reservation algorithms have been proposed to improve QoS. It considers only the handoff to the cell and dose not considers handoff from the cell [6, 11]. In this paper, we propose a new scheme that uses an accurate mobility prediction algorithm to predict MS next location in main road as well as small street and open area and then use this data to predict the expected path of the MS and the expected time for the MS to handoff to and from the cell. We then develop an adaptive bandwidth reservation scheme which considers both incoming and out going call. The reservation algorithm adaptively reserves bandwidth at each BTS according to prediction algorithm to reduce handoff drop rate and improve bandwidth utilization and QoS.

The remainder of this paper is organized as follows. Section II describes the bandwidth reservation technique which we have developed. Section III presents the simulation results. Finally, in section IV we present the conclusion.

\section{DYNAMIC BANDWIDTH RESERVATION ALGORITHM}

In this section we present a Dynamic Bandwidth Reservation Algorithm (DBR) which uses mobility prediction to give priority for handoff call over new call and improves QoS. The algorithm reserves some bandwidth pool for handoff calls. This reserved pool is dynamically adjusted according to the required bandwidth in the BTS and its neighbors. The presented algorithm firstly predicts the MS movement using road map information in main road and shadow cluster method in small streets. For each call the algorithm estimates the arrival and departure time. Finally the bandwidth reservation algorithm estimates the required reserved bandwidth in each cell.

\section{A. Assumption And Mobility Prediction Algorithm}

In our proposed technique we use the traffic flow theory [13 - 15] which is concerned about safe and efficient car and user movement. Traffic flow theory suggests that street system in urban area can be divided into: small street which provides land access with 
small speed, and highway which provides high speed, and collector road which connect small street with high way. Since it's not possible to feed the BTS with a very large number of small streets and intersections, so we assume that the BTS will be equipped with road map which contains main road data (highways and collectors road). In the proposed bandwidth reservation algorithm presented in this paper we use our mobility prediction algorithm [17]. The operation of our mobility prediction algorithm is divided into: Main roads and small streets. In main road, the probability that MS in cell $\mathrm{i}$ to visit cell $\mathrm{j}$ is determined as follows,

$$
\mathrm{P}_{\mathrm{i}, \mathrm{j}}=\sum_{\forall \mathrm{r}} \mathrm{P}_{\mathrm{i}, \mathrm{j} / \mathrm{r}} \cdot \mathrm{P}_{\mathrm{r}}
$$

Where $\mathrm{P}_{\mathrm{r}}$ is the probability that MS takes a specific route ' $\mathrm{r}$ ', $\mathrm{P}_{\mathrm{i}, \mathrm{j} / \mathrm{r}}$ is the probability that the MS in cell 'i' reach cell ' $\mathrm{j}$ ' given that MS takes route ' $\mathrm{r}$ ' and $\forall \mathrm{r}$ are the all routes from cell i to cell $\mathrm{j}$.

In small streets, the probability that MS in cell $\mathrm{i}$ to visit cell $\mathrm{j}$ is determined as follows,

$$
\mathrm{P}_{\mathrm{i}, \mathrm{j}}=\frac{k}{N}
$$

Where $\mathrm{k}$ is the number of points on the elliptic sector which are inside cell $\mathrm{j}, \mathrm{N}$ is the number of all points on the elliptic sector.

Mobility prediction algorithm will be used to determine the probability of certain MS to handoff to certain BTS within next algorithm updating time $\left(\mathrm{T}_{\text {upd }}\right)$. At the same time it will be used to calculate the arrival and departure time of the MS to and from the cell.

\section{B. Estimation Of Arrival And Departure Time.}

In order to get desirable call blocking probability without over reservation of bandwidth, BTS should know the exact time for MS to handoff to and from the BTS and the required bandwidth. Since the BTS dose not know the exact path of the MS and also the time for the MS to handoff to and from it, mobility prediction algorithm is used to predict arrival and departure time of the MS. But if the MS handoff to the BTS occurs before its expected arrival time or handoff from the BTS occurs later than its expected departure time this will increase handoff dropping rate. Therefore to overcome this problem in bandwidth reservation algorithm, we assume outgoing handoff occurs at latest departure time (which is the upper bound for departure time) and incoming handoff occurs at earliest arrival time (which is the lower bound for departure time) as described below.

In main roads, we can calculate the probability that the MS will spend less time than $\mathrm{T}_{\mathrm{ld}}$ (the latest departure time of the MS) before it leaves current cell as follows,

$$
P_{i, j / r}=P\left(\left(\sum_{i=1}^{n} T_{\operatorname{delay}(i)}+\sum_{i=1}^{n+1} d_{i} / v_{i}\right)<\mathrm{T}_{\mathrm{ld}}\right)
$$




$$
\begin{aligned}
& P_{i, j / r}=P\left(\sum_{i=1}^{n} T_{\text {delay }(i)}<\left(\mathrm{T}_{\mathrm{ld}}-\sum_{i=1}^{n+1} d_{i} / v_{i}\right)\right) \\
& \text { let } \frac{T_{l d}-\sum_{i=1}^{n+1} d_{i} / v_{i}-n \mu}{\sigma \sqrt{n}}=\Psi \\
& \int_{0}^{\Psi / \sqrt{2}} \frac{1}{\sqrt{\Pi}} e^{-z^{2}} d z=0.5+\int_{-\infty}^{\Psi} \frac{1}{\sqrt{2 \Pi}} e^{\frac{-y^{2}}{2}} d y \approx \mathrm{P}_{\mathrm{i}, \mathrm{j} / \mathrm{r}} \\
& \mathrm{P}_{\mathrm{i}, \mathrm{j} / \mathrm{r}} \approx 0.5+0.5 \cdot \operatorname{erf}(\psi / \sqrt{2})
\end{aligned}
$$

To get $T_{l d}$, we will use a probability threshold value $P_{\text {th1 }}$ such that $P_{i, j / r} \geq P_{t h 1}$, then

$$
\begin{aligned}
& 0.5+0.5 \cdot \operatorname{erf}(\psi / \sqrt{2}) \geq P_{t h 1} \\
& \frac{T_{l d}-\sum_{i=1}^{n+1} d_{i} / v_{i}-n \mu}{\sigma \sqrt{2 n}} \geq \operatorname{erf}^{-1}\left(2 \cdot\left(P_{t h 1}-0.5\right)\right) \\
& T_{l d} \geq \sum_{i=1}^{n+1} d_{i} / v_{i}+n \mu+\sigma \sqrt{2 n} \cdot \operatorname{erf}^{-1}\left(2 \cdot\left(P_{t h 1}-0.5\right)\right)
\end{aligned}
$$

So we can get $\mathrm{T}_{\mathrm{ld}}$ as,

$$
T_{l d}=\sum_{i=1}^{n+1} d_{i} / v_{i}+n \mu+\sigma \sqrt{2 n} \cdot \operatorname{erf}^{-1}\left(2 \cdot\left(P_{t h 1}-0.5\right)\right)
$$

Similarly, we can calculate the probability that the MS will spend greater time than $T_{\text {ea }}$ (earliest arrival time of the MS) until it enters next cell as follows,

$$
\begin{gathered}
P_{i, j / r}=P\left(\left(\sum_{i=1}^{n} T_{\text {delay }(i)}+\sum_{i=1}^{n+1} d_{i} / v_{i}\right)>\mathrm{T}_{\mathrm{ea}}\right) \\
P_{i, j / r}=P\left(\sum_{i=1}^{n} T_{\text {delay }(i)}>\left(\mathrm{T}_{\mathrm{ea}}-\sum_{i=1}^{n+1} d_{i} / v_{i}\right)\right) \\
\text { let } \frac{T_{e a}-\sum_{i=1}^{n+1} d_{i} / v_{i}-n \mu}{\sigma \sqrt{n}}=\Psi
\end{gathered}
$$




$$
\begin{aligned}
\int_{0}^{\Psi / \sqrt{2}} \frac{1}{\sqrt{\Pi}} e^{-z^{2}} d z=0.5-\int_{-\infty}^{\Psi} \frac{1}{\sqrt{2 \Pi}} e^{\frac{-y^{2}}{2}} d y \approx \mathrm{P}_{\mathrm{i}, \mathrm{j} / \mathrm{r}} \\
\mathrm{P}_{\mathrm{i}, \mathrm{j} / \mathrm{r}} \approx 0.5-0.5 \cdot \operatorname{erf}(\psi / \sqrt{2})
\end{aligned}
$$

To get $\mathrm{T}_{\text {ea, }}$, We will use a probability threshold value $\mathrm{P}_{\mathrm{th} 2}$ such that $\mathrm{P}_{\mathrm{i}, \mathrm{j} / \mathrm{r}} \geq \mathrm{P}_{\mathrm{th} 2}$, then

$$
0.5-0.5 \cdot \operatorname{erf}(\psi / \sqrt{2}) \geq P_{t h 2}
$$

$$
\begin{aligned}
& \sqrt{2} \cdot \operatorname{erf}^{-1}\left(0.5-P_{t h}\right) \geq \frac{T_{l d}-\sum_{i=1}^{n+1} d_{i} / v_{i}-n \mu}{\sigma \sqrt{n}} \\
& T_{e a} \leq \sum_{i=1}^{n+1} d_{i} / v_{i}+n \mu+\sigma \sqrt{2 n} \cdot e^{-1}\left(2 \cdot\left(0.5-P_{t h 2}\right)\right)
\end{aligned}
$$

So we can get $\mathrm{T}_{\mathrm{ea}}$ as,

$$
T_{e a}=\sum_{i=1}^{n+1} d_{i} / v_{i}+n \mu+\sigma \sqrt{2 n} \cdot e^{-1}\left(2 \cdot\left(0.5-P_{t h 2}\right)\right)
$$

On the other hand, in local streets, latest departure time and earliest arrival time of MS can be calculated as follows.

Actually the handoff happens in most cases near the theoretical cell boundary (assume cell radius $\mathrm{R}$ ) with error $\pm \Delta \mathrm{R}$ as shown in fig.1. We will define two borders (imaginary borders) for the cell in addition to the existing border. The inner one of them has radius of $R-\Delta R$, and will be used to calculate the time when the MS handoff to the neighbor cell $\left(T_{\text {ea }}\right)$. The outer has radius of $\mathrm{R}+\Delta \mathrm{R}$ and will be used to calculate the time when the MS handoff from the current cell $\left(\mathrm{T}_{\mathrm{ld}}\right)$.

Earliest arrival time can be calculated as,

$$
\mathrm{T}_{\text {ea }}=\left(\mathrm{d}_{\mathrm{av}} / \mathrm{v}_{\mathrm{av}}\right)
$$

Where $\mathrm{v}_{\mathrm{av}}$ is the average speed of the MS and $\mathrm{d}_{\mathrm{av}}$ is the average distance that MS passed to enternext cell.

For example in fig.1. The average distance for the MS to entercell 1 is the average distance between MS and inner border of cell 3. In this case $d_{a v}$ is, $\mathrm{d}_{\mathrm{av}}=\left(\mathrm{d}_{1}+\mathrm{d}_{2}+\mathrm{d}_{3}\right) / 3$.

Cell 3 will calculate $T_{\text {ea }}$ and sends it to cell 1 . Cell 1 will consider incoming handoff will occur at time $\mathrm{T}_{\text {ea. }}$

Also latest departure time can be calculated as,

$$
\mathrm{T}_{\mathrm{ld}}=\left(\mathrm{D}_{\mathrm{av}} / \mathrm{V}_{\mathrm{av}}\right)
$$


Where $D_{a v}$ is the average distance that MS passed until it leaves current cell.

For example in fig.1. The average distance for the MS to leave cell 3 is the average distance between MS and outer border of cell 3 . In this case $\mathrm{D}_{\mathrm{av}}$ is,

$\mathrm{D}_{\mathrm{av}}=\left(\mathrm{D}_{1}+\mathrm{D}_{2}+\mathrm{D}_{3}\right) / 3$

Cell 3 will consider outgoing handoff will occurs at time $\mathrm{T}_{\text {ld. }}$.

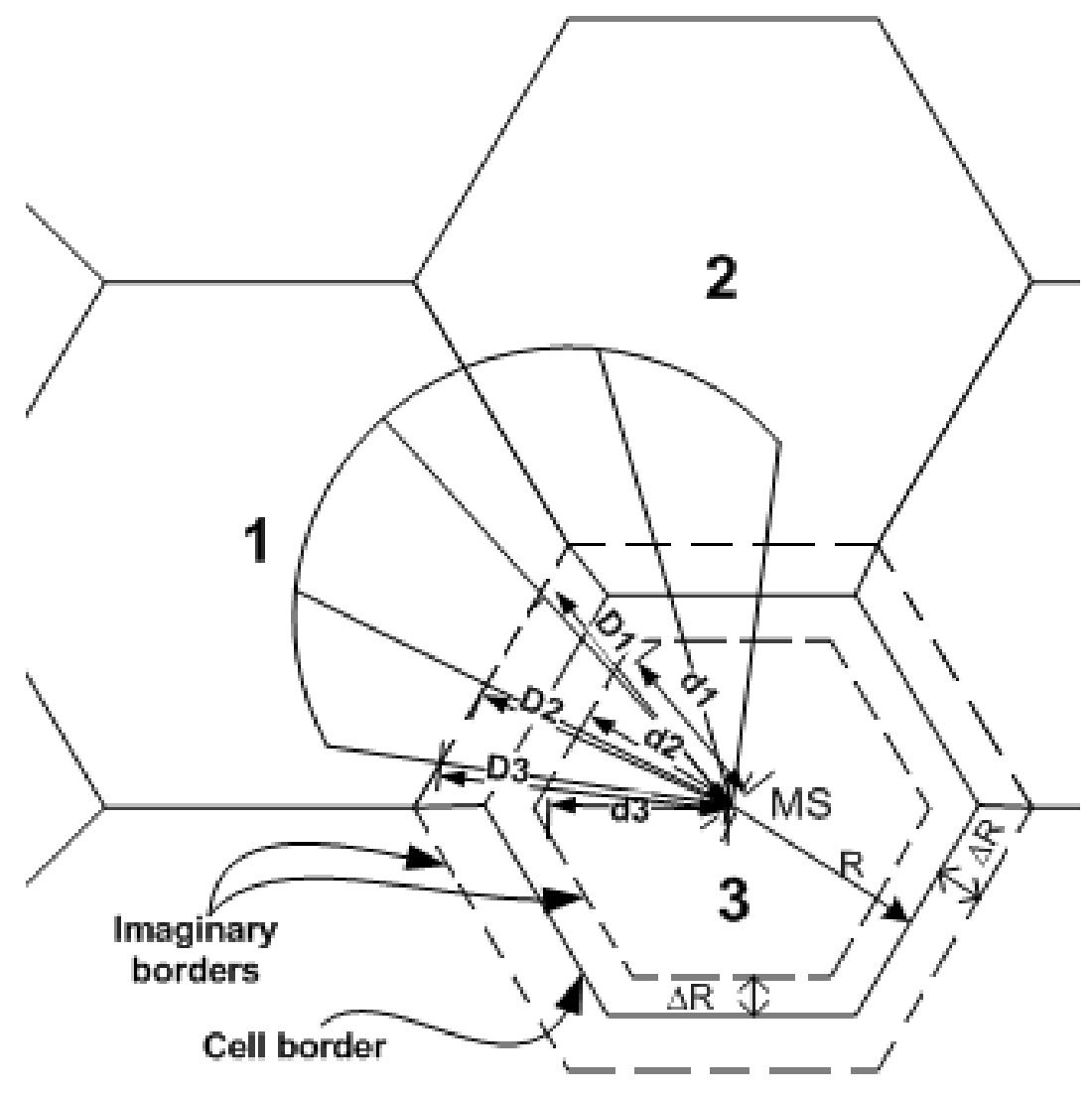

Fig. 1. Calculation of $\mathrm{T}_{\text {ea }}, \mathrm{T}_{\mathrm{ld}}$ in small street

For clarity, we Assume that we have reference cell " cell i " and its neighbor cell " cell $\mathrm{j}$ " the reference cell should do the following procedure to consider incoming handoff and outgoing handoff.

1- Reference cell calculates $T_{l d}$ for MSs currently connected to it and will leave to neighbor cell $\mathrm{j}$ and the probability of visiting the neighbor cell. Reference cell will consider this handoff as outgoing handoff at time $\mathrm{T}_{\mathrm{ld}}$.

2- Reference cell calculates $\mathrm{T}$ ea for MSs currently connected to it and will enterneighbor cell $\mathrm{j}$ and the probability of visiting the neighbor cell, and then it sends these data to neighbor cell. Cell $\mathrm{j}$ will consider this handoff as incoming handoff at time $\mathrm{T}_{\mathrm{ea}}$.

3- Reference cell receives from neighbor cell (cell j) $T_{\text {ea }}$ for MSs currently connected to neighbor cell (cell j) and will handoff to the reference cell and the probability of 
visiting the reference cell. Reference cell will consider this handoff as incoming handoff at time $\mathrm{T}_{\text {ea. }}$

4- Reference cell will do the previous steps for all its neighbor cells.

5- All cells will do as what reference cell does.

\section{C- Bandwidth Reservation}

To give priority for the handoff call over the new call, the BTS reserves some bandwidth for handoff call. This reserved bandwidth is shared for handoff MSs even if it did not do bandwidth reservation in advance. This reserved bandwidth called reservation pool $\mathrm{R}_{\mathrm{p}}$.

To calculate the required reserved bandwidth, each cell considers incoming handoff as increasing of required reserved bandwidth and outgoing handoff as decreasing of required reserved bandwidth. The total required reserved bandwidth changes at each handoff. The total required reserved bandwidth during next $T_{\text {up }}$ is the summation of these handoffs.

As shown in fig 2. We consider incoming handoff as up arrow and outgoing handoff as down arrow. The length of the arrow is equal to the required bandwidth for this handoff multiplied by the probability of this handoff. We can see that the maximum required reserved bandwidth unit $\left(\mathrm{R}_{\mathrm{p}}\right)$ during next algorithm updating time (assume $\mathrm{T}_{\text {upd }}=20 \mathrm{sec}$ ) is 5 unit so $\mathrm{R}_{\mathrm{p}}=5$ bandwidth unit.

The reserved bandwidth in cell $\mathrm{j}$ by neighbor cell $1\left(\mathrm{R}_{\mathrm{plj}}\right)$ can be written as,

$$
\mathrm{R}_{\mathrm{plj}}=\left[\sum_{\forall i} B \cdot P_{i j} U\left(t-T_{e a i}\right)-\sum_{\forall k} B \cdot P_{k l} U\left(t-T_{l d k}\right)\right]
$$

Where $\forall i$ are all MS in cell 1 and will handoff to cell $\mathrm{j}$ at time $\mathrm{T}_{\mathrm{ea}}<\mathrm{T}_{\text {upd, }} \mathrm{P}_{\mathrm{ij}}$ is the probability of $\mathrm{MS}_{\mathrm{i}}$ handoff to cell $\mathrm{j}$ at time $\mathrm{T}_{\mathrm{ea}}, \forall k$ are all $\mathrm{MS}$ in cell $\mathrm{j}$ that will handoff to neighbor cell 1 in time $T_{l d}<T_{u p d}, P_{k l}$ is the probability of $\mathrm{MS}_{\mathrm{k}}$ in cell $\mathrm{j}$ to handoff to cell 1 at time $T_{l d}, B$ is the bandwidth required by the MS (We assume that every MS need one bandwidth unit to make a call) and $\mathrm{U}(\mathrm{t})$ is the unit step function.

The total reserved bandwidth in cell $\mathrm{j}, \mathrm{R}_{\mathrm{p} j}$, is,

$$
\mathrm{R}_{\mathrm{pj}}=\text { Maximum peak of }\left[\sum_{\forall q} R{ }_{p q j}\right]
$$

Where $\forall q$ are all neighbors of cell $\mathrm{j}$.

As mention above, to give priority for handoff call over new call, each BTS should reserve some bandwidth, $R_{p}$, that can only be utilized by handoff call. A new call is accepted in cell $\mathrm{j}$ only if the free remaining bandwidth after accepting the new call is greater than or equal to $\mathrm{R}_{\mathrm{p}}$ i.e.,

$$
C(j)-\left(C_{u}(j)+B_{N}\right) \geq R_{p j}
$$

Where $C(j)$ is the total bandwidth in cell $j, C_{u}(j)$ is the used bandwidth in cell $j, B_{N}$ is the required bandwidth for the new call and $R_{p j}$ is the reserved pool in cell $j$. 
Handoff call is accepted in cell $\mathrm{j}$ if there is sufficient bandwidth for it i.e.,

$$
\mathrm{C}(\mathrm{j})-\left(\mathrm{C}_{\mathrm{u}}(\mathrm{j})+\mathrm{B}_{\mathrm{h}}\right) \geq 0
$$

where $B_{h}$ is the required bandwidth for the handoff call.

Finally we can summarize the goal of our algorithm as, we give priority for handoff call over new call by reserving some bandwidth, $\mathrm{R}_{\mathrm{p}}$, that can only be utilized by handoff call. This bandwidth is dynamically changed based on mobility prediction algorithm used. As a result we can get low handoff drop rate without over reserving the resources. This will result in better bandwidth utilization and low new call drop rate.

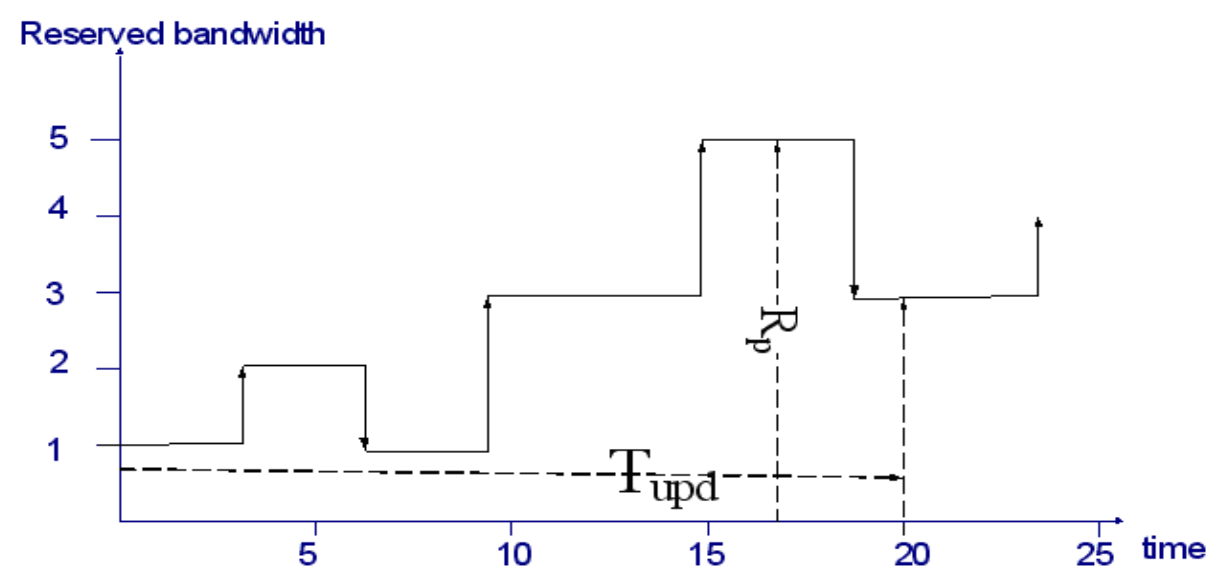

Fig.2. The reserved bandwidth Rp.

\section{SIMULATION RESULTS}

The performance measurement has been made by comparing our proposed scheme (DBR) with Road Map [6] and elliptic curve [11] in terms of new calls block rate, handoff calls drop rate and bandwidth utilization.

\section{Mobility Model}

In this paper we use Gauss-Markov mobility model to simulate user's mobility. The Gauss-Markov model represents a wide range of mobility patterns, including, as its two extreme cases, the random-walk and the fluid-flow models [16]. Therefore the future location of MS can be calculated based on previous MS's location using GaussMarkov model. Recursive realization of Gauss-Markov model can be written as [16],

$$
\begin{aligned}
& v x(t)=\alpha \cdot v x(t-1)+(1-\alpha) \mu x+\sigma x \sqrt{1-\alpha^{2}} N x \\
& v y(t)=\alpha . v y(t-1)+(1-\alpha) \mu y+\sigma y \sqrt{1-\alpha^{2}} N y
\end{aligned}
$$

Where $\mathrm{vx}$ is the MS's speed component in $\mathrm{x}$ direction and $\mu \mathrm{x}$ its mean and $\sigma \mathrm{x}$ its standard deviation, vy is the MS's speed component in y direction and $\mu \mathrm{y}$ its mean 
and $\sigma \mathrm{y}$ its standard deviation, $\mathrm{N}$ is the normally distributed random variable with zero mean and unit variance and $\alpha$ is the memory level.

We assume that stationary user remains stationary during call holding time, and moving user can be divided into two groups: pedestrian and low speed users in small street and high speed users in main roads. We modulate the MS's motion using GaussMarkov model. If the MS's location in main road, the MS in this case will follow the road and can turn only at the intersections with predefined turn probability, and the MS's speed depends on the MS's average speed and road average speed. Memory level in this case is high ( $\alpha$ near 1$)$ which mean that the MS's motion nears fluid-flow model. If the MS isn't in main road (inside local street or open area) We modulate the MS's motion using Gauss-Markov model with $\alpha$ near 0, which mean that the MS's motion nears random-walk.

We assume that the MS can start call at any location in the cellular network which consists of identical hexagon cells. The MS is connected to the nearest BTS. The simulation area is circular if the MS go out of left side it will reenter from right side. Our prediction algorithm updates the MS's speed every $\mathrm{T}_{\text {upd. }}$. Unlike previous algorithm [2] which assumes that the MS moves in constant speed during call holding time.

We assume that cell size is $1.5 \mathrm{~km}$, number of cells $\mathrm{m}=19$, all cells are the same size, the MS's mean speed in main road is in the rang of 60 to $120 \mathrm{~km} / \mathrm{h}$, in local street in the rang of 10 to $70 \mathrm{~km} / \mathrm{h},\left|\phi_{0}\right|=\pi / 16$, probability of meeting a red traffic light $P_{\text {red }}=0.4$, mean delay time of red traffic light $\mu_{\text {red }}=35 \mathrm{sec}, \mathrm{P}_{\mathrm{th} 1}=\mathrm{P}_{\mathrm{th} 2}=0.7,\left(\mathrm{P}_{\mathrm{th}} \mathrm{can}\right.$ be used to dynamically change the reservation pool), variance delay time of red traffic light $\sigma_{\text {red }}^{2}=15 \mathrm{sec}$ and algorithm updating time $\mathrm{T}_{\text {upd }}=20 \mathrm{sec}$.

\section{Traffic Model}

It was assumed that the arrival rate for new calls is a Poisson distribution with a mean of $\lambda$. The call holding time is exponentially distributed with mean $1 / \mu$. The number of channels in each cell is a constant $\mathrm{C}$. The normalized offered traffic load of the system is defined to be,

$$
\mathrm{L}=\frac{\lambda}{m \cdot \mu_{c} \cdot C}
$$

Where $\mathrm{m}$ is the number of cells in the system and the load is measured in Erlang.

\section{Performance and result}

We present simulation results for the new call blocking probability $\left(\mathrm{P}_{\mathrm{ncb}}\right)$ which mean that new call block due to the lack of bandwidth in current cell, handoff dropping probability $\left(\mathrm{P}_{\mathrm{hd}}\right)$ which men that the call drop during handoff from one cell to neighbor cell due to lack of bandwidth in neighbor cell. In addition we will represent the results for bandwidth utilization which mean the usage of the total bandwidth in each cell. This is calculated as the ratio of all used bandwidth to all the bandwidth for that cell. The remaining part of the bandwidth is composed of reserved and available bandwidth that is not currently used. It is said to be worse performance that the bandwidth utilization is low while the system is heavily loaded because the utilization should 
grow with system load in a optimal condition. Finally we will compare our algorithm with Road Map (RM) algorithm and elliptic curve algorithm.

First we assume that all MS that move in the network are in the road that equipped in the road map. From fig. 3, we find that, our algorithm (DBR) performs better than RM

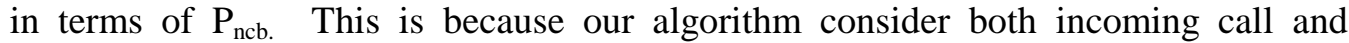
outgoing call so it reserve less resources which result in more bandwidth available for new call. Also we find that RM gives better result than elliptic curve that's because elliptic curve didn't consider the delay time at road intersection so it reserves more resource.

From fig. 4, we find that the three algorithms give low handoff drop rate (less than 0.005). In fig.5, we find that our algorithm gives better bandwidth utilization than RM and elliptic curve. That is because our algorithm can correctly estimate the required reserved bandwidth. Also we find that RM give better bandwidth utilization than elliptic curve. This is because elliptic curve reserve more resources than required.

Since road map only contains the main road data. So RM algorithm will be less efficient if MSs move in small street or open area. This will increase $\mathrm{P}_{\text {hd }}$. For example if $8 \%$ of the MSs move in small street the result will be as follow.

In fig. 6 , we find that the proposed algorithm give better result in terms of new call blocking rate than RM. Also RM gives better result than elliptic curve since elliptic curve reserve more resources than required.

From fig. 7, we find that elliptic curve algorithm gives a little bit better results in terms of handoff drop rate than our algorithm, while our algorithm gives better results than RM. Since our algorithm use an accurate mobility prediction algorithm for MS motion in main road as well as small street, it can correctly estimate the required bandwidth. This lead to reduce $\mathrm{P}_{\text {hd }}$ without over reserving the resources so that we can get better bandwidth utilization as shown in fig.8. In this figure we find that the proposed algorithm gives better bandwidth utilization than road map and road map gives better result than elliptic curve.

We can notice that both values of $\mathrm{P}_{\text {ncb }}$ and $\mathrm{P}_{\text {hd }}$ obtained when the traffic load is 0.6 or less are very close to each other for all the algorithms examined. Therefore, we may have a low handoff drop rate even without using mobility prediction algorithm.

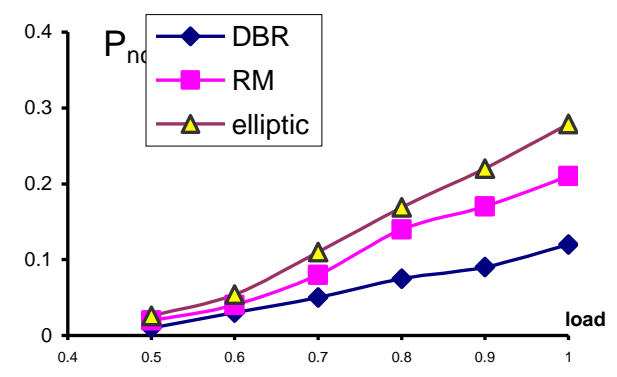

Fig. 3. System load versus $\mathrm{P}_{\text {ncb }}$

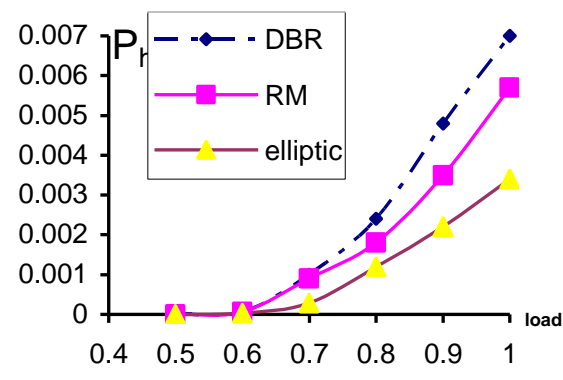

Fig.4. System load versus $\mathrm{P}_{\text {hd }}$ 


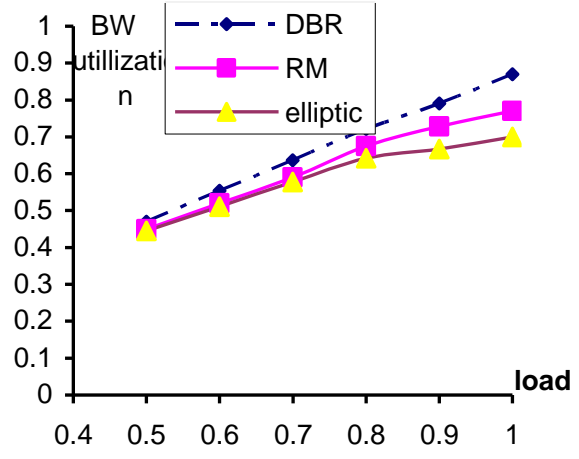

Fig.5. System load versus BW utilization

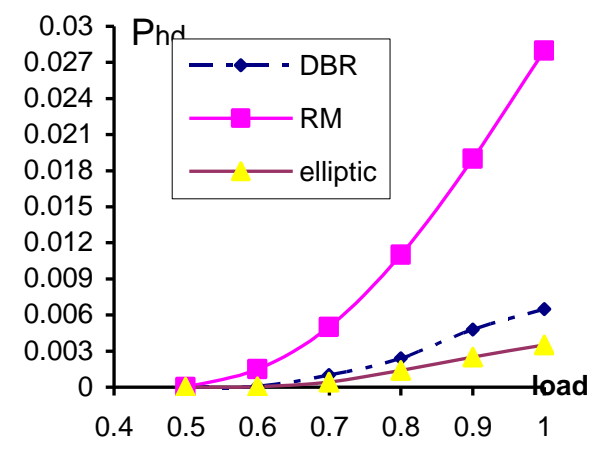

Fig.7. System load versus $P_{\text {hd }}$ ( $8 \%$ out of the road)

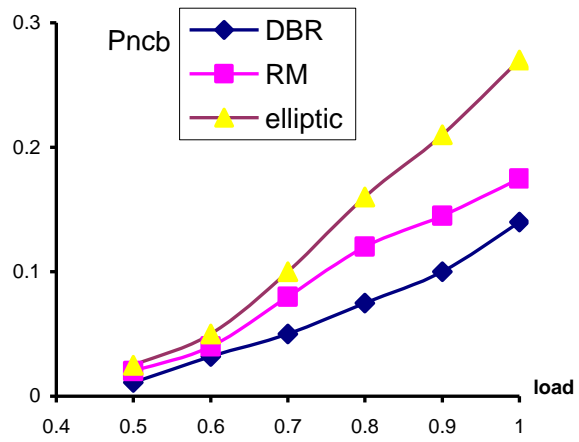

Fig.6. System load versus $\mathrm{P}_{\text {ncb }}(8 \%$ out of the road)

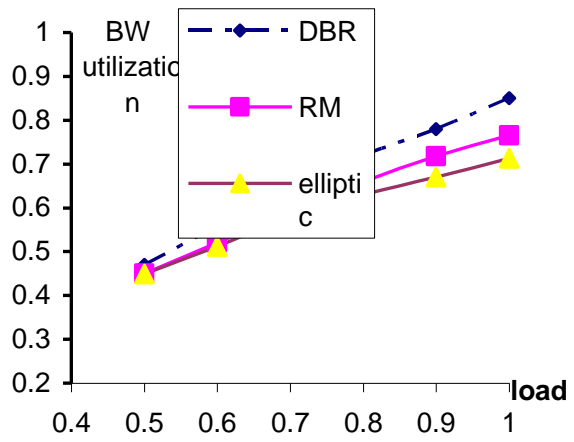

Fig.8. System load versus BW utilization ( $8 \%$ out of the road)

\section{CONCLUSION}

In this paper, we propose a dynamic bandwidth reservation algorithm (DBR) for wireless networks. The DBR uses an accurate mobility prediction scheme that is suitable for MS motion prediction in main road as will as small street. Among the many possible applications where mobility prediction can be used, we use it for handoff prioritization. The algorithm predicts the expected path of the MS as well as the expected time for the MS to handoff to and from the cell, these prediction values are used to determine the required bandwidth that should be reserved in each cell. This reserved bandwidth is dynamically changing depending on MS mobility. The proposed reservation algorithm considers both incoming and outgoing calls. The goal of our reservation algorithm is to reduce handoff drop rate without over reserving the bandwidth for handoff so that we can get better bandwidth utilization. We compared our reservation algorithm with similar algorithms, Road Map and Elliptic Curve. Simulation results show that our algorithm reduce handoff drop rate at the same time it gives better bandwidth utilization and better probability of new call block rate. 


\section{REFERENCES}

[1] N.D. Tripathi, J.H. Reed and H.F. VanLandinoham; "Handoff in cellular systems" IEEE Pers. Commun. , Vol. .5, No.6, pp. 26-37 Dec. 1998.

[2] D. Hong and S.S. Rappaport; "Traffic model and performance analysis for cellular mobile radio telephone systems with prioritized and non-prioritized handoff procedures" IEEE Trans. on Veh. Tech., pp. 77-92 Aug. 1986.

[3] D. Levine, I. Akyildiz and M. Naghshineh, "A Resource estimation and call admission algorithm for wireless multimedia networks using the shadow cluster concept,"IEEE/ACM Trans. On Networking, Vol. 5, pp 1-12, Feb.1997.

[4] F. Yu and V. Leung "Mobility-based predictive call admission control and bandwidth reservation in wireless cellular networks," proceedings-IEEEINFOCOM .v1, (IEEE cat no 01CH 37213) pp 518-526, 2001.

[5] Y.C. Kim, D.E. Lee; Dynamic channel reservation based on mobility in wireless ATM networks” IEEE Communications Magazine, pp. 47-51 Nov. 1999.

[6] D. Lee, Y. Hsueh, "Bandwidth reservation scheme based on road information for next generation cellular networks,"IEEE TRANSACTION ON VEHICULAR NETWORK Vol. 53, No. 1, pp. 243-252, January 2004.

[7] M.H. Chiu and M.A. Bassiouni; "Predictive schemes for handoff prioritization in cellular networks based on mobile positioning" IEEE Journal on Selected Areas in Communications, Vol.18, No.3, pp. 510-522, March 2000.

[8] Z.Xu, Z. Ye, S.V. Krishnamurthy, S.K. Tripathi and M. Molle "A New adaptive channel reservation scheme for handoff calls in wireless Cellular networks" Proceedings of the Second International IFIP-TC6 Networking Conference on Networking Technologies, Services, and Protocols; Performance of Computer and Communication Networks; and Mobile and Wireless Communications, pp. 672 - 684, May, 2002 .

[9] S. Choi and K. G. Shin, "Predictive and adaptive bandwidth reservation for handoffs in QoS-sensitive cellular networks," in Proceedings of ACM SIGCOMM'98, pp. 155-166, Sept. 1998.

[10] W. Soh and H. S. Kim, "Dynamic Bandwidth Reservation in Cellular Networks Using Road Topology Based Mobility Predictions, Infocom 2004.

[11] X. Zhou, Ruiliang, C. Gao "An elliptical shadow algorithm for motion prediction and resource reservation in wireless cellular networks ", proceeding of 10th Int. Conference on computer communication and Networks, Scottsdele, Arizona, USA,pp. 234-239, Oct. 2001.

[12] M. M. Islam, M. Murshed and L. S. Dooley, "A novel Mobility Support Resource Reservation and Call Admission Control Scheme for Quality-ofService Provision in Wireless Multimedia Communications" ICC 2004 - IEEE International Conference on Communications, vol. 27, no. 1, pp. 48-52, June 2004.

[13] W. R. McShane and R. P. Roess, " Traffic engineering," Prentice Hall Polytechnic Series in Traffic Engineering, 1990.

[14] J. G. Markoulidaskis, G. L. Lyberopoulos, D. F. Tsirkas and E. D. Sykas "Mobility modeling in third-generation mobile telecommunication systems" IEEE personal communication, vol. 4, no. 4, pp. 41-56, August 1997. 
[15] Y. Bejerano, I. Cidon , "Efficient location management based on moving location areas," IEEE INFOCOM 2001 - The Conference on Computer Communications, no. 1, pp. 3-12, April 2001.

[16] B. Liang, and Z.J.Haas, "Predictive distance-based mobility management for multidimensional PCS networks," IEEE/ACM Trans. On Networking, VOL. 11, NO. 5,pp.718-732, Oct. 2003.

[17] I. I. Ibrahim, A. S. Ali, A. F. Ghanim, "Mobility prediction Algorithm For wireless Networks" JES Assiut University, Voi. 34, NO. 1, PP. 199-213, January 2006. 